\title{
La modelación matemática: un análisis de los planteamientos en documentos curriculares colombianos
}

\section{Mathematical modeling: an analysis of approaches in curricular documents in Colombia}

SARMIENTO-RIVERA, Dermin ${ }^{1}$

ALDANA, Eliecer ${ }^{2}$

SOLAR, Horacio ${ }^{3}$

\begin{abstract}
Resumen
Este documento tiene como propósito confrontar la fundamentación teórica sobre modelación en los documentos curriculares colombianos (MEN, 1998; 2006) con las tareas sugeridas para su implementación en los DBA (MEN, 2016) en torno a la formación para el ejercicio de valores democráticos. Las clasificaciones de Font (2002), Da Silva y Kato (2012) y Villa-Ochoa, Castrillón-Yepes y Sánchez-Cardona (2017) permiten consolidar el objetivo. De otra parte, la metodología cualitativa posibilitó esbozar discrepancias y oportunidades de incorporación de la modelación con una perspectiva crítica.

Palabras clave: modelación matemática crítica, documentos curriculares, valores democráticos, empoderamiento crítico
\end{abstract}

\begin{abstract}
The purpose of this document is to confront the theoretical foundation on modeling in the Colombian curricular documents (MEN, 1998; 2006) with the tasks suggested for their implementation in the DBA (MEN, 2016) around the training for the exercise of democratic values. The classifications of Font (2002), Da Silva and Kato (2012) and Villa-Ochoa, Castrillón-Yepes and Sánchez-Cardona (2017) allow to consolidate the objective. On the other hand, the qualitative methodology made it possible to outline discrepancies and opportunities for incorporating the modeling with a critical perspective.

Key words: Critical mathematical modeling, curriculum documents, democratic values, critical empowerment.
\end{abstract}

\section{Introducción}

Dentro de las investigaciones sobre el currículo de matemáticas se documentan dos problemáticas que involucran las decisiones del profesor, una es la inclusión de los problemas de la realidad y otra tiene que ver con la práctica curricular en las instituciones. En el primer caso, la preocupación se centra en el papel de las aplicaciones y modelación matemática en los currículos de matemáticas (Niss y Højgaard, 2011; Burkhardt,

\footnotetext{
${ }^{1}$ Estudiante de Doctorado en Ciencias de la Educación, Universidad del Quindío. Magíster en Ciencias de la Educación, Universidad de la Amazonia. Licenciado en Matemáticas y Física, Universidad de la Amazonia. Contacto: derminsarmiento@gmail.com

2 Doctor en Educación Matemática, Universidad de Salamanca, España. Magister en Administración de la Educación, Énfasis en Dirección, Universidad del Valle. Licenciado en Matemáticas, Universidad del Quindío. Contacto: eliecerab@uniquindio.edu.co

${ }^{3}$ Doctor en Didáctica de las Matemática, Universitat Autónoma de Barcelona. Magíster en Didáctica de las Ciencias Experimentales y de las Matemáticas, Universitat Autónoma de Barcelona. Magíster en Historia de las Ciencias, Universitat Autónoma de Barcelona. Licenciado en Matemáticas, Pontificia Universidad Católica de Chile. Contacto: hsolar@uc.cl
} 
2006). Mientras que, en el segundo caso, el foco está en la interpretación, reflexión y toma de decisiones de los profesores frente a los documentos curriculares nacionales e institucionales (Gimeno-Sacristán, 1995, Gómez y Velazco, 2017).

En ambos casos, se presenta una relación dinámica entre el nivel de prescripción del currículo y el nivel de moldeado del currículo implementado por las instituciones y el evaluado por el profesor. Para Gimeno-Sacristán (1995), en el nivel de prescripción predominan los cruces de múltiples prácticas e intereses de campos sociales (políticos, económicos, educativos, científicos, entre otros) que estructuran un currículo nacional. Mientras que en el nivel de moldeado del currículo el papel central está en las reflexiones epistemológicas y ontológicas de los profesores. En este nivel curricular es fundamental la reflexión del profesor, por lo cual Perrenoud (2007) habla de la necesidad de reflexionar en la acción y sobre la acción misma para transformarla. En el campo particular de la educación matemática, D’Amore (2005) considera que las reflexiones del profesor sobre las matemáticas y la educación matemática dirigen las elecciones sobre los propósitos formativos y el diseño de oportunidades de aprendizaje y movilización de competencias que orientaran su práctica.

La importancia de reflexionar sobre estos documentos radica en el hecho que son el insumo ofrecidos por el Ministerio de Educación en Colombia para orientar la práctica curricular y pedagógica. Sin embargo, como mencionan Gómez y Velazco (2017), estos documentos son usados parcialmente por los profesores en ejercicio, es más, los autores concluyen que "En el caso de los ejemplos [en los DBA], no es claro que un profesor de primaria o la mayoría de los de secundaria estén en capacidad de incluir los ejemplos en su práctica de aula, si así lo deciden" (p. 278). De manera simular, Villa-Ochoa, Bustamante, Berrio, Osorio y Ocampo (2008) documentan que en las prácticas de aula persiste una visión de las matemáticas infalibles y acabadas, posición que dista por mucho de los nuevos propósitos formativos de la educación matemática principalmente del ejercicio de valores democráticos.

Por otra parte, diferentes autores reconocen que la modelación matemática es un vehículo para promover diferentes aspectos de la crítica, por ejemplo, (Da Silva y Kato, 2012) identifican en la literatura entre los aspectos que contribuyen a este propósito: la participación crítica y democrática en el aula de clases, implementación de acciones comunitarias y análisis de las estructuras de poder en la sociedad. Al respecto, Villa-Ochoa (2010) reporta que, pese a estas ventajas, las propuestas y prácticas curriculares están basadas "en un desarrollo rígido de contenidos y en el desarrollo de habilidades de tipo procedimental en los estudiantes, se han mostrado como limitantes para el desarrollo de procesos de modelación" (p. 168). En consecuencia, el centro de la discusión en este documento es develar hasta qué punto los documentos curriculares, en lo relacionado con la modelación matemática, promueven prácticas que están a espaldas del ejercicio de valores democráticos.

\subsection{Educación matemática y el sujeto político}

Al pensar en la formación matemática para el ejercicio de valores democráticos es propicio cuestionar lo que entendemos por valores democráticos y cuál es el aporte de los diferentes enfoques en educación matemática a este propósito de formación. En el primer caso, Carrillo (2011) plantea que la educación democrática y para la democracia tiene por objetivo ofrecer herramientas para una vida más humana, justa y pacífica, en este sentido considera que en la escuela se debe promover y fortalecer actitudes y valores democráticos. En este plano de la vida humana, la autora genera tres grupos de valores democráticos, el primero corresponde a la vida humana como desarrollo ético, en el cual agrupa: la justicia, la paz y el civismo; en el segundo grupo relacionado con el compromiso responsable ubica la libertad y la diferencia; en el tercer grupo, el derecho a tener derecho, es constituido por: la igualdad, la solidaridad, el respecto y la cooperación. Así mismo, para Skovsmose y Valero (2012), vinculado con la democracia como acción política subyace el valor de la participación ciudadana, íntimamente relacionada con ideas matemática poderosas desde el punto de vista cultural, en la cual es 
indispensable empoderar a los sujetos políticos con herramientas de protección ciudadana y lucha social entre las cuales se encuentran: el derecho de petición, la acción de tutela, acciones populares, acciones de grupo.

Por otra parte, la construcción de currículos de matemáticas está asociado con un enfoque teóricos particular de lo que son las matemáticas, en este plano, de la investigación en didáctica de las matemáticas, existen algunas tipificaciones del cuerpo teórico construido, en una de estas clasificaciones Font (2002) identifica siete programas de investigación, a saber: el enfoque cognitivo, el constructivismo radical, el constructivismo social, el enfoque sistémico, el enfoque antropológico, el enfoque semiótico y el enfoque crítico. Por otra parte, también es posible identificar tres virajes, el primero fue denominado por D’Amore (2005) como el viraje antropológico, aludiendo a la Teoría Antropológica de lo Didáctico- TAD (Chevalard, 1999). Está teoría, se desplaza de una visión ontológica realista a una pragmática, en la cual, los objetos matemáticos existen en tanto que las personas reconozcan que existen. Pero tal reconocimiento, es posible en la medida que, las personas se relacionen con fenómenos en una actividad semiótica de la cual emergen los objetos en forma de sistemas de representación a través de un proceso de construcción de praxeologías estructuradas en: tareas, técnicas, tecnologías y teoría.

En relación con la formación para la ciudanía, aunque en la construcción de praxeologías se considera el contexto, éste tiene el papel de proveer un entorno de construcción de conceptos en el aula, por lo tanto, es posible conectar esta propuesta con ideas matemáticas fundamentadas en la lógica y en la ideología de la certeza. Autores como Borba y Skovsmose (2003); Skovsmose (1999); Skovsmose y Valero (2012) reconocen que estas ideas suponen que para el ejercicio de la ciudadanía y los valores democráticos es suficiente el dominio de las matemáticas, desconociendo que la democracia lejos de ser algo alcanzado es más un ideal en el cual es indispensable el compromiso de las personas en la coflexión, debate y transformación de prácticas que se escapan del salón en las que el sujeto está inmerso.

El segundo giro, según Lerman (2000) es el viraje hacia lo social. En este marco, Valero (2012a) considera que el conocimiento, el razonamiento y el significado, surgen del "encuentro entre seres humanos históricamente anclados, contextualizados, y su actividad en ámbitos y espacios particulares que están estructurados socialmente" (p. 202). Las investigaciones instaladas en este viraje, consolidan varias vertientes entre ellas la educación matemática crítica- EMC (Lerman, 2000; Planas, 2010; Valero, Andrade-Molina y Montecino, 2015; Valero, 2012a; Skovsmose, 1999; Alrø, Ole y Valero, 2010), esta vertiente, centra su foco en el cuestionamiento de la neutralidad de las matemáticas en la sociedad, es decir reconoce que las matemáticas históricamente han aportado al progreso, pero también han causado estragos (Valero, 2012a; Skovsmose, 1999).

En particular, la EMC orienta su actuar investigativo en tres vías "la crítica a las matemáticas en la sociedad; la relación entre educación matemática y democracia, justicia social, equidad e in(ex)clusión; y la invención de nuevas posibilidades educativas." (Valero et al., 2015, p. 10). Llama la atención la segunda línea de investigación porque relaciona diferentes elementos considerados en los documentos de curriculares colombianos, por ejemplo: competencias, valores democráticos y la idea de una educación matemática para todos. En este sentido, Skovsmose (1999) plantea que para el ejercicio de la democracia es indispensable la competencia matemática crítica, la cual vincula al estudiante como sujeto político con la posibilidad de cuestionar el uso de las matemáticas en la sociedad y las decisiones de los gobernantes, es decir con la posibilidad de crítica social. De esta manera, el investigador cuestiona la idea de alfabetización ingenua en la que se supone que la mera apropiación de un contenido matemático permite, per se, ejercer la democracia.

El tercer viraje, el político, está relaciona directamente con la EMC, pero acentúa la mirada en el uso que hace el estado- nación sobre la educación para constituirla en una maquinaria para la construcción de subjetividades que contribuyan a sus fines económicos y políticos (Valero, 2017; Valero et al., 2015). 
Valero (2017) considera que, pensar en la educación matemática para formar sujetos con valores democráticos como la justicia social, la equidad, el ejercicio de la crítica ante las formas de poder, entre otras, requiere que tal formación contribuya al empoderamiento de las personas. Sin embargo, cuestiona la forma en que se usa el término y propone dos significados, el empoderamiento intrínseco y el empoderamiento crítico. El primero de ellos, propone que "la abstracción, la axiomatización, la formalización y el carácter verdadero de las matemáticas" (Valero, 2017, p. 106) son atributos que forman mejores capacidades de pensamiento y por lo tanto es posible ejercer la participación ciudadana.

El empoderamiento crítico, por otra parte, ubica el foco en "poder ver más allá de los hechos para pensar en las decisiones que se toman diariamente con base en tales modelos expertos matemáticos" (Valero, 2017, p. 108). Esto se traduce, en la posibilidad, de los sujetos políticos, de tomar distancia de aquellas prácticas sociales que implican un riesgo social, pero como son camufladas en modelos matemáticos es indispensable develar las intenciones de sometimiento social. En tal caso, los modelos matemáticos, en el aula, se convierten en un vehículo para obstaculizar o para promover la crítica social y el ejercicio de la democracia en general.

Este último aspecto, relaciona la modelación matemática y el sujeto político (estudiante y profesor) con la necesidad de pensar nuevas prácticas educativas. En este sentido, las tareas y ambientes de modelación juegan un papel importante en la participación de los sujetos en la movilización de aprendizajes y competencias matemáticas. En consecuencia, a continuación, se presentan algunos desarrollos de la modelación matemática, especialmente la perspectiva crítica.

\subsection{Modelación Matemática}

Un punto de referencia sobre el estudio de la modelación matemática en educación matemática lo ofrece la International Community of Teachers of Mathematical Modelling and Aplications - ICTMA. Esta comunidad fue conformada en 1983 con el propósito de investigar la influencia de la modelación matemática y las aplicaciones en la enseñanza y aprendizaje de las matemáticas (Biembengut y Hein, 2013). Más de 30 años después, la investigación en el campo ha crecido y se ha diversificado. Así, Kaiser y Sriraman (2006) identifican múltiples perspectivas de la modelación: (1) modelización realista o aplicada, (2) modelización contextual, (3) modelización educativa, (4) modelación sociocrítica, (5) modelación epistemológica o teórica y (6) la modelación cognitiva como meta-perspectiva.

Otra clasificación es presentada en Erbaş y otros (2014) y en Stillman (2015), en las cuales, se identifican dos enfoques diferentes en este dominio, a saber: Como objetivo o fin de la enseñanza y Como medio o vehículo. Como objetivo, fin o propósito, se considera que la modelación matemática es un proceso o competencia matemática que se desarrolla a lo largo del proceso educativo. Mientras que en la noción de vehículo la modelación se enfoca en la enseñanza de conceptos y procedimientos matemáticos.

Considerando, que la intención es develar como la modelación matemática, tal como se plantea en los documentos curriculares colombianos, contribuye a la formación para la democracia, se amplía en la perspectiva crítica de este dominio para posteriormente considerar el papel de las tareas y ambientes de modelación en la movilización de los propósitos formativos.

\subsection{Perspectiva crítica de la modelación matemática}

Esta perspectiva es planteada inicialmente por Barbosa $(2003 ; 2006)$ el autor considera que las decisiones sociales se basan en modelos matemáticos y que sus propósitos por lo general están ocultos al público, en este sentido el modelo matemático, como producto del proceso de modelación, es una herramienta que ayuda a los estudiantes a ejercer la ciudadanía crítica cuando devela ideologías y hegemonías. 
En esta misma perspectiva Orey y Rosa (2007) plantea la noción de eficacia socio-critica como un propósito de la educación en general y de la educación matemática en particular. Esta noción propone que la modelación matemática es una metodología que permite a los estudiantes identificar fuerzas represivas y opresivas que pueden vivir en diferentes niveles de la sociedad para luego ejercer acciones de liberación mediante la construcción de un discurso racional que se fundamente en modelos emancipadores.

En esta perspectiva de la modelación, Araújo (2009) propone que esta perspectiva de la modelación matemática, se preocupe por promover la participación critica de los estudiantes/ciudadanos en aspectos sociales, políticos, económicos, entre otros. La autora, considera, al igual que Skovsmose (1999) que los proyectos son indispensables para abordar este tipo de participación en el aula de matemáticas.

En una revisión de literatura sobre la modelación matemática en la perspectiva sociocrítica, Da Silva y Kato (2012) concluyen que es posible identificar custro grandes características configuradas por unidades de significados (ver tabla 1).

Tabla 1

Características de la modelación crítica

\begin{tabular}{|c|c|}
\hline Característica & Unidad de significado \\
\hline $\begin{array}{l}\text { Participación activa de los } \\
\text { estudiantes en la construcción del } \\
\text { modelo }\end{array}$ & $\begin{array}{l}\text { Trabajo en grupo; } \\
\text { Participación crítica y democrática en las clases; } \\
\text { Elección del problema por parte de los alumnos. }\end{array}$ \\
\hline $\begin{array}{l}\text { Participación activa del estudiante en } \\
\text { la sociedad }\end{array}$ & $\begin{array}{l}\text { Desarrollo de acciones comunitarias } \\
\text { Extensión al contexto social } \\
\text { Desempeño crítico en la sociedad } \\
\text { Importancia de las matemáticas en la sociedad. }\end{array}$ \\
\hline $\begin{array}{l}\text { Estudio de problemas no } \\
\text { matemáticos de la realidad }\end{array}$ & $\begin{array}{l}\text { Usar problemas no matemáticos de la realidad; } \\
\text { Elección de problemas por parte de los estudiantes; } \\
\text { interpretar modelos matemáticos según la realidad; } \\
\text { considerar la cultura estudiantil; } \\
\text { Importancia de las matemáticas en la sociedad }\end{array}$ \\
\hline El papel del profesor como mediador & $\begin{array}{l}\text { Trabajo en grupo; } \\
\text { Elección del problema por parte de los alumnos; } \\
\text { participación crítica y democrática en el aula; } \\
\text { Considerar la cultura estudiantil; } \\
\text { Importancia de las matemáticas en la sociedad. }\end{array}$ \\
\hline
\end{tabular}

Fuente: Da Silva y Kato (2012)

Valero (2002; 2006; 2017) y Camelo et al., (2013) dejan ver otra característica relacionada con la necesidad de considerar al estudiante como un sujeto político que ha decidido no aprender matemáticas porque considera que, dadas sus condiciones de desventaja social (inequidad, pobreza, violencia, injusticia social, por enunciar algunas), no tiene sentido aprenderlas. Bajo esta mirada, la modelación puede ser un vehículo para empoderar críticamente a las personas en diversas situaciones de riesgo social si en el aula son estudiadas prácticas sociales "que atañen a su bienestar y el de su comunidad" (Valero, 2017, p. 110). En este sentido, en Camelo, Mancera y Perilla (2017) llaman la atención sobre la necesidad de reflexionar sobre cómo abordar las realidades de los estudiantes en el aula.

\subsection{Tareas de modelación}

Las características de la modelación matemática en el enfoque crítico permiten proponer una reflexión sobre los tipos de tareas que se deberían proponer para movilizar este proceso en la educación básica y media en Colombia. En relación a esta reflexión, Villa-Ochoa et al., (2017) plantean una clasificación alternativa compuesta 
por: enunciados verbales, construcción de representaciones, modelación a través de proyectos y uso y análisis de modelos. Esta tipificación muestra una amplia gama de andamios para la construcción de oportunidades de movilización de la modelación en la construcción de planes de aula en diferentes niveles educativos, sin embargo, de acuerdo con esta clasificación los problemas de palabras auténticos y los proyectos y análisis de modelos proporcionan ambientes en los que la modelación se convierta en una herramienta para ejercer la ciudadanía responsable, más allá de permitir la construcción conceptos matemáticos.

Los problemas de palabras auténticos consideran las prácticas sociales de una comunidad para ser comprendidas a través de las matemáticas (Muñoz Mesa, Londoño Orrego, Jaramillo y Villa-Ochoa, 2014). Ampliando sobre la autenticidad, Strobel, Wang, Weber y Dyehause (2012) distinguen cuatro focos de ambientes autentico: contextos, tareas, de impacto y de valor. De manera similar, Los proyectos involucran las situaciones auténticas con diferentes intenciones entre ellas fomentar la crítica sobre el uso de las matemáticas en la sociedad y proponer alternativas de solución, Sin embargo, Camelo, Perilla y Mancera (2016) consideran importante promover estrategias como los recorridos para involucrar a los estudiantes en la identificación de problemáticas sociales. Por último, en términos del empoderamiento crítico, el sujeto político es el encargado de cuestionar también los modelos usados en la sociedad y usarlos como instrumento de lucha social.

\section{Metodología}

Este documento, es uno de los productos del estudio de doctorado del autor principal, en el cual, el objetivo principal de la investigación fue comprender los aspectos que configuran la práctica curricular usada para movilizar la competencia matemática modelizar crítica. El estudio se llevó a cabo en una institución rural ubicada en el departamento del Huila-Colombia. La fase trabajo de campo de esta investigación cualitativa estuvo centrada en un proceso de formación, implementado entre febrero y noviembre de 2019 y diseñado con la estructura del ciclo de investigación - acción propuesto por Elliot (1994) y Kemmis y McTaggart, R. (1988), basado en ciclos de reflexión, planeación, implementación, observación y análisis. De esta manera, cada sesión se organizaba en: bases para la reflexión, diseño de la práctica, acuerdos para la implementación y consolidación de la autonomía.

Aquí se reporta, sobre el análisis que suscito la pregunta ¿qué criterios deberíamos tener en cuenta para diseñar las oportunidades de aprendizaje, de donde provienen y cuál es el papel de la modelación en este diseño? Esta pregunta constituyo la base para la reflexión de la segunda y tercera sesión. Como respuesta, se acordó, de acuerdo con Ñaupas, Valdivia, Palacios y Romero (2014) llevar a cabo el análisis documental, teniendo en cuenta que en esta técnica "no es el estilo del texto que se pretende analizar, sino las ideas expresadas en él" (p. 391). Esta técnica fue aplicada a: los lineamientos curriculares-LC (MEN, 1998), los Estándares Básicos de Competencias-EBC (MEN, 2006) y los Derechos Básicos de Aprendizaje-DBA (MEN, 2016), en torno a dos aspectos, el primero sobre los fundamentos de los nuevos principios de formación y el segundo, sobre los fundamentos de modelación matemática.

Para llevar a cabo el análisis de los documentos, se diseñó para el primer aspecto, una matriz en torno a tres aspectos de la perspectiva de la educación matemática crítica contenidos en lo que Font (2002) y Valero (2000) identificaron como la relevancia de las matemáticas escolares, a saber: problemas de la vida real, participación ciudadana y cuestionamiento de las decisiones. En el caso del análisis de los fundamentos sobre modelación se usa la clasificación propuesta en Kaiser y Sriraman (2006) y en el análisis de la tarea la clasificación de Villa-Ochoa et al. (2017) ubicando el foco en los enunciados verbales. 


\section{Resultados}

\subsection{Hacia una perspectiva crítica de la Educación Matemática en el currículo colombiano}

En el campo curricular, la reflexión realizada a partir de preguntas como ¿qué matemáticas enseñar y cómo enseñarlas? Motivó algunas reformas en diferentes países, entre ellos Colombia, donde en 1978 se inició un proceso llamado Renovación Curricular, en el que se propuso "la elaboración de un marco teórico global que permitiera precisar los criterios con los cuales se deberían hacer la revisión y el diseño de los programas de los nueve grados de la educación básica" (MEN, 1998 b, p. 16).

Como respuesta a las preguntas anteriores, los encargados de la reforma tomaron postura sobre el enfoque el sistémico como plataforma para levantar en 1994, mecanismos de evaluación curricular y la organización del sistema educativo con la formulación de la Ley General de Educación (ley 115 de 1994). Este proceso conlleva a formular los Lineamientos Curriculares de Matemáticas (MEN, 1998) y los Estándares Básicos De Competencias en Lenguaje, Matemáticas, Ciencias Y Ciudadanía (MEN, 2006) documentos que forman el currículo oficial colombiano, denominados referentes de calidad. En estos documentos, Colombia como muchos otros países construyeron un currículo conformado por el cruce entre contenidos, procesos y contextos, es decir, adoptó un currículo por competencias, el cual requirió formular nuevos factores o propósitos para la educación matemática escolar.

En este plano, de los nuevos factores que sustentan la inclusión de las matemáticas escolares en el currículo, se encontró un direccionamiento de la educación matemática en los enfoques sociocultural y crítico, bajo esta mirada podría decirse que lo propósitos formativos encuadran en el giro social de la educación matemática. En primer lugar, sociocultural, porque ubica la actividad matemática en la resolución de problemas de la realidad; segundo, el enfoque crítico aparece por la inclusión de los valores democráticos vinculados con en la educación matemática ofrece la oportunidad de una participación directa en la transformación de prácticas de riesgo.

Es posible identificar una visión sociocultural de las matemáticas, en el sentido que proponen Bishop (2005) y Valero (2012b) como práctica sociocultural que tiene que ver con la interacción en el aula y con el uso del contexto como fuente de prácticas que sirven de andamio para construir matemáticas y para cuestionar el uso de las matemáticas y particularmente de los modelos matemáticos en la sociedad.

En el caso de los Estándares Básicos de Competencias EBC (MEN, 2006) existe una tendencia al enfoque crítico, específicamente por la inclusión de tres nuevos factores de la formación matemática "la necesidad de una educación básica de calidad para todos los ciudadanos, el valor social ampliado de la formación matemática y el papel de las matemáticas en la consolidación de los valores democráticos" (MEN, 2006. p. 47).

El primer factor, una educación básica de calidad para todos los ciudadanos, atiende al reconocimiento de la equidad, inclusión y cobertura, este factor hace frente a la brecha labrada entre clases sociales, raza y género que actuaron y que pueden actuar como filtro en la educación matemática, como presenta Skovsmose y Niss (2004) la educación matemática puede coartar el ejercicio de los valores democráticos de diferentes formas por ejemplo: “(1) discriminación en términos de (falta de) recursos, (2) racismo, (3) sexismo, (4) discriminación en términos de lenguaje, (5) la discriminación en términos de lo que se conoce como 'habilidad', y (6) el desempoderamiento a través de la descomposición" (p.13). En este plano, actúa el concepto de disonancia al reconocer que "las matemáticas están fuertemente asociadas con la creación de estructuras de riesgo en la nuestra sociedad actual" (Valero, 2006, p. 10). 
Tabla 2

Perspectiva crítica de la educación matemática en los documentos curriculares colombianos.

\begin{tabular}{|c|c|}
\hline Característica & Fragmento del documento \\
\hline \multirow{3}{*}{$\begin{array}{l}\text { Problemas de la vida } \\
\text { real }\end{array}$} & $\begin{array}{l}\text { El conocimiento es considerado como una actividad social que debe tener en } \\
\text { cuenta los intereses y la afectividad del niño (MEN, 1998, p. 29). }\end{array}$ \\
\hline & $\begin{array}{l}\text { Cuando hablamos de la actividad matemática en la escuela destacamos que el } \\
\text { alumno aprende matemáticas haciendo matemáticas, lo que supone como } \\
\text { esencial la resolución de problemas de la vida diaria" (MEN, 1998, p. 97). }\end{array}$ \\
\hline & $\begin{array}{l}\text {... se hace necesario comenzar por la identificación del conocimiento matemático } \\
\text { informal de los estudiantes en relación con las actividades prácticas de su entorno } \\
\text { y admitir que el aprendizaje de las matemáticas no es una cuestión relacionada } \\
\text { únicamente con aspectos cognitivos, sino que involucra factores de orden afectivo } \\
\text { y social, vinculados con contextos de aprendizaje particulares (MEN, 2006, p. 47) }\end{array}$ \\
\hline \multirow{4}{*}{$\begin{array}{l}\text { Participación } \\
\text { ciudadana }\end{array}$} & $\begin{array}{l}\text { La segunda razón alude al conocimiento matemático imprescindible y necesario } \\
\text { en todo ciudadano para desempeñarse en forma activa y crítica en su vida social } \\
\text { y política y para interpretar la información necesaria en la toma de decisiones } \\
\text { (MEN, 2006, p. 48) }\end{array}$ \\
\hline & $\begin{array}{l}\text {...para participar en la preparación, discusión y toma de decisiones y para } \\
\text { desarrollar acciones que colectivamente puedan transformar la sociedad. (MEN, } \\
2006, \text { p. 48). }\end{array}$ \\
\hline & $\begin{array}{l}\text { Este factor agrega a las demás funciones de la formación matemática una nueva } \\
\text { función política: la preocupación por la formación en valores democráticos y por } \\
\text { el ejercicio de la ciudadanía crítica (MEN, 2006, p. 48). }\end{array}$ \\
\hline & $\begin{array}{l}\text {...es necesario que en los procesos de enseñanza de las matemáticas se asuma la } \\
\text { clase como una comunidad de aprendizaje donde docentes y estudiantes } \\
\text { interactúan para construir y validar conocimiento, para ejercer la iniciativa y la } \\
\text { crítica y para aplicar ese conocimiento en diversas situaciones y contextos (MEN, } \\
2006, \text { p. 48). }\end{array}$ \\
\hline $\begin{array}{l}\text { Cuestionamiento de } \\
\text { decisiones }\end{array}$ & $\begin{array}{l}\text { hay que hacer énfasis en los actos comunicativos, de tal suerte que se le permita } \\
\text { al grupo deliberar sobre las razones o la falta de ellas, sobre las conjeturas, } \\
\text { opiniones o juicios y sobre las ventajas o desventajas de las posibles decisiones } \\
\text { que deban tomarse dentro y fuera de la clase y que tengan resonancia colectiva } \\
\text { (MEN, 2006, p. 48). }\end{array}$ \\
\hline
\end{tabular}

Fuente: Construcción propia.

Pese al buen propósito, enmarcado en el primer factor, los lineamientos curriculares después de reconocer el papel de los intereses y necesidades de los niños como aspectos sustanciales de la enseñanza y el aprendizaje, manifiesta como papel central del profesor de matemáticas vigilar el dominio de ciertas prácticas matemáticas, porque estas "son una herramienta intelectual potente, cuyo dominio proporcionan privilegios intelectuales y ventajas intelectuales" (MEN, 1998, p. 29). Este discurso, se acerca más a la noción de ideas matemáticas poderosas basadas en la lógica, el cual da por sentado que las habilidades matemáticas garantiza el acceso a mejores condiciones de vida. Claramente esta postura induce la discriminación por habilidad y pone en la palestra la posibilidad de proveer las condiciones de igualdad entre los sujetos.

De manera similar, el segundo factor, El valor social ampliado de la formación matemática hace especial énfasis en reconocer la necesidad de reflexionar sobre la complejidad de sociedad actual y el papel que deberían ejercer las matemáticas en el ejercicio de la ciudadanía critica. En estos términos, el segundo factor, abona terreno a las ideas matemáticas poderosas en la visión sociológica, en la cual Skovsmose y Valero (2012) llama la atención sobre que "las matemáticas operan como una parte integral de muchas acciones tecnológicas y que tales acciones, como cualquier otra, pueden tener consecuencias positivas o negativas impredecibles" (p. 46). 
El tercer factor, el papel de las matemáticas en la consolidación de valores democráticos, evidencia otro aspecto del enfoque crítico de la educación matemática, que plantea el papel de las matemáticas en la formación de ciudadanos democráticamente competentes, es decir reconoce la dimensión política de la educación matemática y de los aprendices ciudadanos. Esta dimensión política, según Valero (2002); Skosvmose (1999); Alvis, Aldana y Solar (2019) destaca la necesidad de concebir el aula y la escuela como escenarios para el ejercicio valores democráticos mediante la participación de los estudiantes como sujetos políticos en situaciones de su vida especialmente llama la atención sobre despertar la sensibilidad por las necesidades del otro y de emprender acciones de transformación social conjunta. En este sentido, la modelación matemática y las matemáticas mismas pueden actuar como herramientas de emancipación crítica, siempre y cuando exista una estrecha vinculación con las ideas matemáticas poderosas desde el punto de vista cultural.

En síntesis, los Estándares Básicos de Competencia y los lineamientos curriculares muestran la inclusión de fines sociales, culturales y políticos, lo cual constituye una tendencia al enfoque crítico de la educación matemática. Esta tendencia vislumbra, la necesidad de considerar al estudiante y al profesor como sujetos políticos que ejercen una competencia matemática crítica mediante la participación directa sobre asuntos de injusticia o desigualdad social, presentes en su comunidad (Valero, 2002; 2017). Sin embargo, desde este punto de vista, se cuestiona la visión de ideas matemáticas poderosas desde la lógica oculta la oportunidad de vincular prácticas matemáticas democratizantes, en el sentido que las matemáticas no son inherentemente democráticas.

\subsection{La modelación matemática como vehículo para la participación ciudadana}

La modelación matemática cobra importancia en la estructura curricular al presentarse como uno de los procesos generales de las matemáticas, estos procesos generales constituyen una propuesta para cambiar a una enseñanza de las matemáticas que supere el enciclopedismo y que fomente el desarrollo el desarrollo de competencias (MEN, 2006; Díaz-Barriga, 2011). Este análisis busca reflexionar sobre la coherencia entre la postura crítica de la educación matemática que presentan en los referentes de calidad y las propuestas de movilización de la modelación matemática.

En la tabla 3 se presentan algunos fragmentos de los referentes de calidad, lineamientos y estándares de competencia, con el fin de identificar los enfoques que se promueven sobre el proceso general modelación matemática.

En los lineamientos curriculares (MEN, 1998) proceso de modelación matemática se formula en conexión con la resolución de problemas relacionados con aspectos de la vida diaria del estudiante, por esta razón propone la modelación como la actividad de construir modelos matemáticos pasando de la realidad a las matemáticas y de nuevo a la realidad. Sin embargo, en los fragmentos presentados, se nota un marcado énfasis en la fase de trabajo matemático, con el fin último de generalizar.

Lo anterior es consistente con lo analizado en Villa-Ochoa y Ruiz-Vahos (2009) cuando concluyen que los ejemplos formulados en los lineamientos curriculares, están configurados en contextos intra-matemáticos y realidades ficticias (Alsina, 2007), lo cual se opone a la perspectiva pragmática de las matemáticas. Principalmente estas situaciones distanciadas de la realidad y demasiado simplificadas sirven para poder introducir o desarrollar un concepto, pero descuidan la comprensión de la realidad, el cuestionamiento de las prácticas socioculturales y la posibilidad de llevar el proceso de modelación en el aula como propósito formativo. 
Tabla 3

Enfoques de la modelación en los referentes de calidad

\begin{tabular}{|c|c|c|}
\hline Documento & Fragmento del documento & Enfoque \\
\hline \multirow[t]{2}{*}{$\begin{array}{l}\text { Lineamientos } \\
\text { curriculares }\end{array}$} & $\begin{array}{l}\text { "La resolución de problemas en un amplio sentido se considera siempre en conexión con } \\
\text { las aplicaciones y la modelación. La forma de describir ese juego o interrelación entre el } \\
\text { mundo real y las matemáticas es la modelación" (MEN, 1998, p. 11). }\end{array}$ & Realista \\
\hline & $\begin{array}{l}\text { La modelación es un proceso muy importante en el aprendizaje de las matemáticas, que } \\
\text { permite a los alumnos observar, reflexionar, discutir, explicar, predecir, revisar y de esta } \\
\text { manera construir conceptos matemáticos en forma significativa. (MEN, 1988, p. 80) }\end{array}$ & Realista \\
\hline \multirow[t]{3}{*}{$\begin{array}{l}\text { Estándares } \\
\text { Básicos de } \\
\text { competencias }\end{array}$} & $\begin{array}{l}\text { [la modelación] como simplificación y restricción de la complejidad de una situación real } \\
\text { para reducirla a una situación ya conocida, de tal manera que se pueda detectar } \\
\text { fácilmente qué esquema se le puede aplicar, cómo se relaciona con otras y qué } \\
\text { operaciones matemáticas pueden ser pertinentes para responder a las preguntas que } \\
\text { suscita dicha situación (MEN, 2006, p. 52) }\end{array}$ & Realista \\
\hline & $\begin{array}{l}\text { las matemáticas que va más allá de la descripción usual de ellas como la ciencia del } \\
\text { espacio y el número: considera que las matemáticas parten de una base empírica, pero } \\
\text { para detectar en ella esquemas que se repiten, que podemos llamar "modelos" o } \\
\text { "patrones" ("patterns"), y en la multitud de esos modelos o patrones detectar de nuevo } \\
\text { otros más y teorizar sobre sus relaciones para producir nuevas estructuras matemáticas } \\
\text { (MEN, 2006, p. 52) }\end{array}$ & Cognitiva \\
\hline & $\begin{array}{l}\text { Un modelo se produce para poder operar transformaciones o procedimientos } \\
\text { experimentales sobre un conjunto de situaciones o un cierto número de objetos reales o } \\
\text { imaginados, sin necesidad de manipularlos o dañarlos, para apoyar la formulación de } \\
\text { conjeturas y razonamientos y dar pistas para avanzar hacia las demostraciones. (MEN, } \\
\text { 2006, p. 52) }\end{array}$ & Cognitiva \\
\hline
\end{tabular}

Fuente: Construcción propia a partir de MEN (1998; 2006)

En este sentido, existe un predominio de la modelación como vehículo para la construcción de conceptos de forma significativa. En esta forma de usar la modelación, el enfoque realista de la modelación en la visión de Gravemeijer (2007), promueve la movilización el uso de las situaciones del mundo real o situaciones contextuales, en muchos casos hipotéticas o ficticias, con el fin de promover la construcción de modelos básicos que van formalizándose hasta una estructura matemática que pueda ser aplicada para resolver el problema. En coherencia con este uso, Julie (2002) plantea que la modelación como vehículo "se ve como la búsqueda de una fórmula para describir la situación bajo investigación" (p. 4). Principalmente, esto corresponde a una tradición en la enseñanza de las matemáticas en la que se les solicita a los estudiantes a identificar patrones basados en fórmulas asociadas a un concepto matemático (Alsina, 2007; Erbaş et al., 2014; Julie, 2002).

Una consecuencia de esta forma de usar la modelación, consiste dejar en el estudiante la idea del contexto como algo innecesario, ya que no se reflexiona nuevamente sobre la validez del modelo en la realidad o esta se centra en la revisión del trabajo matemático subyacente. Este hecho, permite cuestionar las oportunidades de acercamiento al contexto y al reconocimiento de prácticas de riesgo, socialmente relevantes para el ciudadano actual o del futuro. Siguiendo a Da Silva y Kato (2012) la modelación como vehículo, restringe la perspectiva crítica de la modelación y la formación de valores democráticos porque impide el desarrollo de acciones comunitarias, la extensión al contexto social y el desempeño crítico en la sociedad, esenciales para el ejercicio de la ciudadanía crítica.

También, esto es evidente en las matrices de los estándares, en estas la modelación está más asociada al pensamiento variacional y sistemas analíticos (ver tabla 4). En estos estándares prevalece la presencia del trabajo matemático, por ejemplo, en los estándares $(4,5,6,7)$ se induce a profundizar en la fase de trabajo matemático mediante descripciones de variaciones, correlación, establecimiento del tipo de proporcionalidad, 
funciones y derivadas. Así mismo, el uso de la modelación que se plantea es la generalización y el trabajo al interior de las matemáticas. Solo en los estándares $(6,8)$ se menciona el proceso completo de modelación, pero sigue orientándose a la construcción de conceptos matemáticos, dejando de lado el cuestionamiento de las situaciones y prácticas socioculturales de riesgo.

Tabla 4

Selección de estándares relacionados con la modelación matemática

\begin{tabular}{|c|c|c|c|c|}
\hline \multicolumn{5}{|c|}{ Grupos de grados } \\
\hline $1^{\circ}-3^{\circ}$ & $4^{\circ}-5^{\circ}$ & $6^{\circ}-7^{\circ}$ & $8^{\circ}-9^{\circ}$ & $10^{\circ}-11^{\circ}$ \\
\hline \multirow[t]{4}{*}{$\begin{array}{l}\text { Reconozco y describo } \\
\text { regularidades y patrones } \\
\text { en distintos contextos } \\
\text { (numérico, geométrico, } \\
\text { musical, entre otros). (1) }\end{array}$} & $\begin{array}{l}\text { Analizo y explico } \\
\text { relaciones de } \\
\text { dependencia entre } \\
\text { cantidades que varían } \\
\text { en el tiempo con cierta } \\
\text { regularidad en } \\
\text { situaciones } \\
\text { económicas, sociales y } \\
\text { de las ciencias } \\
\text { naturales. (2) }\end{array}$ & $\begin{array}{l}\text { Describo y represento } \\
\text { situaciones de variación } \\
\text { relacionando diferentes } \\
\text { Representaciones. (4) }\end{array}$ & $\begin{array}{l}\text { Modelo situaciones de } \\
\text { variación con } \\
\text { funciones polinómicas. } \\
\text { (6) }\end{array}$ & $\begin{array}{l}\text { Modelo situaciones de } \\
\text { variación periódica con } \\
\text { funciones } \\
\text { trigonométricas e } \\
\text { interpreto y utilizo sus } \\
\text { derivadas. (8) }\end{array}$ \\
\hline & $\begin{array}{l}\text { Predigo patrones de } \\
\text { variación en una } \\
\text { secuencia numérica, } \\
\text { geométrica o gráfica. } \\
\text { (3) }\end{array}$ & & & \\
\hline & & & & $\begin{array}{l}\text { Interpreto la noción de } \\
\text { derivada como razón de } \\
\text { cambio y como valor de } \\
\text { la pendiente de la } \\
\text { tangente a una curva y } \\
\text { desarrollo métodos } \\
\text { para hallar las derivadas } \\
\text { de algunas funciones } \\
\text { básicas en contextos } \\
\text { matemáticos y no } \\
\text { matemáticos.(9) }\end{array}$ \\
\hline & & $\begin{array}{l}\text { Analizo las propiedades } \\
\text { de correlación positiva y } \\
\text { negativa entre } \\
\text { variables, de variación } \\
\text { lineal o de } \\
\text { proporcionalidad } \\
\text { directa y de } \\
\text { proporcionalidad } \\
\text { inversa en contextos } \\
\text { aritméticos y } \\
\text { geométricos. (5) }\end{array}$ & $\begin{array}{l}\text { Identifico relaciones } \\
\text { entre propiedades de } \\
\text { las gráficas y } \\
\text { propiedades de las } \\
\text { ecuaciones } \\
\text { algebraicas. (7) }\end{array}$ & \\
\hline
\end{tabular}

Fuente: MEN (2006)

Entrando en el análisis de las tareas matemáticas propuestas para movilizar la modelación matemática, se identificó que en los Derechos Básicos de Aprendizaje-DBA (MEN, 2016) también son restringidos aquellos que tratan de la modelación. A continuación, es analizado El DBA 4 del grado décimo (Ver tabla 5), el cual plantea un enunciado sobre modelación asociado al estándar "Modelo situaciones de variación periódica con funciones trigonométricas e interpreto y utilizo sus derivadas" (MEN, 2006, p.89). 
Tabla 5

DBA 4, Grado 10

DBA

funciones para

modelar fenómenos

periódicos y justifica

las soluciones.

Evidencia de aprendizaje

1. Reconoce el significado de las razones trigonométricas en un triángulo rectángulo para ángulos agudos, en particular, seno, coseno y tangente.

2. Explora, en una situación o fenómeno de variación periódica, valores, condiciones, relaciones o comportamientos, a través de diferentes representaciones.

3. Calcula algunos valores de las razones seno y coseno para ángulos no agudos, auxiliándose de ángulos de referencia inscritos en el círculo unitario.

4. Reconoce algunas aplicaciones de las funciones trigonométricas en el estudio de fenómenos diversos de variación periódica, por ejemplo: movimiento circular, movimiento del péndulo, del pistón, ciclo de la respiración, entre otros.

5. Modela fenómenos periódicos a través de funciones trigonométricas.

Fuente: (MEN, 2016)

Las evidencias de aprendizaje, en tabla 5, muestran una secuencia de demandas cognitivas asociadas a la modelación como vehículo para la solución de problemas, en este caso centrado en la construcción del concepto de razón trigonométrica y variación periódica. En esta secuencia de evidencias está presente la lógica de aplicación, la cual consiste en partir de la definición de un concepto (evidencia 1) hasta su aplicación en diferentes contextos (evidencia 5). Así mismo, la secuencia corresponde a un enfoque cognitivo de la educación matemática y de la modelación ya que se centra en el uso de las representaciones matemáticas para desarrollar habilidades matemáticas relacionadas con el dominio matemático, la cual niega la posibilidad de develar problemas de la realidad, especialmente aquellos que involucran las necesidades particulares de los sujetos.

En la tarea asociada a este DBA (Figura 1) se puede observar esta lógica y el uso de situaciones hipotéticas asociadas a las evidencias 3 y 4 , que buscan encontrar resultados numéricos y representaciones tabulares o gráficas.

Figura 1

DBA 4 del grado décimo

Construye un disco de radio $12 \mathrm{~cm}$ con diferentes colores, de tal forma que cada franja de color se encuentre a una distancia determinada con respecto al centro del disco. El primer color (azul claro) se encuentra desde el centro del disco hasta un radio de $5 \mathrm{~cm}$ y los demás colores tienen un ancho de un centímetro.

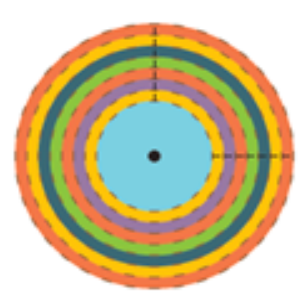

Representa en un plano cartesiano el movimiento que realiza una marca que se hace en algunos de las franjas del disco, cuando éste se hace girar. El centro del disco de colores está en $(0,0)$. Determina los tiempos en los que la marca gira $30^{\circ}$ más a partir de su posición de inicio $\alpha=0^{\circ}$ y realiza la gráfica para estas dos variables hasta una vuelta completa del disco.

Fuente: (MEN, 2016, p. 76)

Esta situación se encuentra en el contexto intra-matemático y de las ciencias físicas, relacionado con el movimiento circular con el cual se espera que los estudiantes construyan y usen el concepto de razón y función trigonométrica. Sin embargo, en la literatura sobre modelación matemática existen algunos trabajos relacionados con problemas de palabras auténticos asociados a contextos culturales que permiten acercarse al mismo concepto, Por ejemplo, Berrio (2011) además de promover la construcción de modelos matemáticos, considera importante comprender las prácticas socioculturales de sembrado del café en terrenos con diferentes, de esta interacción con el contexto emergen las razones trigonométricas. Otro ejemplo en esta perspectiva, lo presentan los profesores que participaron en esta investigación con el siguiente enunciado: 
Figura 2

Situación de modelación

\section{¿La densidad de siembra y el arreglo espacial del cultivo de café con el que cuenta la institución son los apropiados para obtener una producción óptima?|}

Fuente: profesores de la institución educativa Nuestra Señora del Socorro, Pital - Huila

La situación presentada en la figura 2, deviene de la solicitud del comité de cafeteros de renovar el cultivo institucional de café por la edad. Este escenario fue oportuno para solicitar a los estudiantes que argumentaran las razones por las cuales debía cambiar o no el cultivo. Se decidió con los estudiantes que uno de los criterios para una producción optima era una densidad de siembra adecuada, por lo cual se plantea que a partir de la pregunta en la figura 2, argumentaran ante la rectora que debían hacer con el terreno.

Siguiendo la clasificación de tareas de modelación propuesta por Villa-ochoa et al. (2017) la situación de la densidad de siembra, corresponde a un enunciado verbal autentico de impacto, en cual el estudiante enfrenta evidencias de aprendizaje relacionadas con: identificar variables, construir un modelo real (construcción de un croquis, segmentar el terreno en triángulos, tomar medidas, reconstruir el croquis a escala), trabajar con las matemáticas (construir planteamientos trigonométricos) y plantear un modelo matemático (densidad de área del cultivo de café).

Siguiendo la propuesta de Da Silva y Kato (2012) se encontró que la situación vincula estrechamente la participación activa del estudiante mediante el trabajo en grupo y la autonomía en clase; el estudio de problemas no matemáticos de la realidad, particularmente, considerar la cultura de los estudiantes, al considerar aspectos de la práctica sociocultural de siembra y cálculo de la producción de café en el contexto local, los estudiantes deben realizar consultas sobre la práctica de medición del área de terrenos, entre las que encontraros, dividirlo en triángulos desde un punto. Así mismo el papel mediador del profesor hace posible indagar la práctica de medición de áreas, encontrar un vínculo con las razones y los teoremas trigonométricos, pero además contrastar información con los criterios del comité de cafeteros y decidir acerca de lo más conveniente para una buena cosecha dadas las condiciones de densidad de siembra del terreno.

De manera especial se resalta la participación activa del estudiante en la sociedad. En esta situación, el profesor solicitó a los estudiantes construir una carta argumentando la decisión que debería tomar la institución frente al terreno, este hecho, permite la participación ciudadana de los estudiantes, ya que la institución les permitió realizar las adecuaciones necesarias para el mejoramiento de la producción, lo que implicó un contacto con el comité de cafeteros del municipio para llegar a acuerdos. Bajo estas condiciones, la situación se convierte en una propuesta de proyecto que tomó un periodo académico de 8 semanas para abordarlo. De esta manera las evidencias de aprendizaje se amplían al uso de las matemáticas en la sociedad.

Por lo anterior, la situación planteada posibilita incorporar la modelación como vehículo para la participación ciudadana, más que para introducir conceptos, para comprender el uso de las matemáticas y para cuestionar prácticas socioculturales, por ejemplo, en discusiones entre profesores, estudiantes y comité de cafeteros se cuestionaba que los préstamos concedidos a los cafeteros solo se concedían con la densidad de siembra y la variedad de café establecida por ellos. Aquí las evidencias de aprendizaje se relacionan con el cuestionamiento de las decisiones que toman los entes de poder que devienen en algún tipo de injusticia para favorecer intereses particulares. 


\subsection{Modelación matemática crítica como propósito: una alternativa de transformación del currículo}

Otro sentido asignado a la modelación, consiste en considerarla como propósito o como contenido transversal del currículo. Este sentido de la modelación matemática en una perspectiva crítica, implica la construcción de modelos matemáticos a partir de la comprensión de fenómenos naturales, sociales, políticos, económicos sin la condición preexistente de llegar a ciertos conceptos o procedimientos matemáticos como el resultado del proceso de modelación, además implica el escrutinio, disección, crítica, extensión y adaptación de modelos existentes con el objetivo de comprender los mecanismos de uso en la sociedad, como lo expresa Skovsmose (1999), los modelos matemáticos instados por el poder legislativo operan casi siempre de espaldas a los intereses del pueblo, en consecuencia los ciudadanos de manera colectiva deben discernir los interés y advertir los riesgos de tales usos para emprender acciones de transformación.

Sin embargo, en las comprensiones de Carrillo (2011) y Valero (2012b), la democracia lejos de ser un bien alcanzado, es un bien deseado. En relación con lo expuesto en el párrafo anterior, esto quiere decir que acceder a lo democrático en el dominio de la modelación matemática y en general en educación matemática, requiere movilizar tres tipos de arenas: el salón de clases, la organización escolar y la sociedad local y global. Ubicando la mirada en la organización escolar, es necesario cuestionar "Quién participa en la formulación de un currículo y cómo se implementa tal formulación" (Valero, 2012b, p. 52). Tal cuestionamiento, es necesario para empoderar al profesor en la organización de un currículo que le de vida a la modelación, esto es, movilidad y continuidad.

Tabla 6

Continuidad curricular del Proceso de Modelación Matemática

\begin{tabular}{cl}
\hline Documento & \multicolumn{1}{c}{ Fragmento del documento } \\
\hline $\begin{array}{c}\text { Lineamientos } \\
\text { curriculares }\end{array}$ & $\begin{array}{l}\text { se considera que todos los alumnos necesitan experimentar procesos de } \\
\text { matematización que conduzcan al descubrimiento, creación y utilización de } \\
\text { modelos en todos los niveles (MEN, 1998, p. 80) }\end{array}$ \\
\cline { 2 - 3 } & $\begin{array}{l}\text { También, que la variedad de alumnos en la clase puede hacer que se } \\
\text { produzcan diferentes clases de modelos que estén al alcance de los diferentes }\end{array}$ \\
& niños según su desarrollo (MEN, 1998, p. 80) \\
\hline Estándares & $\begin{array}{l}\text { En una situación problema, la modelación permite decidir qué variables y } \\
\text { competencias } \\
\text { relaciones entre variables son importantes, lo que posibilita establecer } \\
\text { modelos matemáticos de distintos niveles de complejidad, a partir de los } \\
\text { cuales se pueden hacer predicciones, utilizar procedimientos numéricos, } \\
\text { obtener resultados y verificar qué tan razonable son éstos respecto a las } \\
\text { condiciones iniciales. (MEN, 2006, p. 53) }\end{array}$ \\
\hline
\end{tabular}

Fuente: construcción propia a partir de MEN (1998; 2006).

En estos fragmentos de la tabla 6, dos expresiones resaltan la continuidad de la modelación a lo largo del proceso educativo. La primera, la utilización de modelos en todos los niveles, hace referencia al desarrollo a largo plazo de los procesos generales (Solar, 2009; Solar et al., 2014), lo cual permite pensar en la necesidad de diseñar planes de aula, unidades didácticas, tareas o ambientes de modelación a partir del análisis de las prácticas socioculturales que generen oportunidades de movilización de la modelación a lo largo del proceso educativo (primaria, secundaria y universidad). La segunda, modelos matemáticos de distintos niveles de complejidad permite reflexionar sobre las diversas posibilidades de procesos, modelos, reflexiones, intereses de los estudiantes por comprender y transformar tanto contextos como prácticas.

Pese a estas intenciones de continuidad de la modelación en el aula, como puede verse en la progresión longitudinal (tabla 4 en la sesión 3.1) de la modelación está centrada en la formalización de conceptos matemáticos concebidos de antemano por el profesor, esto limita las posibilidades organizar planes flexibles que 
permitan el ejercicio de los valores democráticos ya que encasilla y limita las vías de construcción de modelos matemáticos diversos y su extensión a otros modelos más completos, así como la participación activa del estudiante en la sociedad, al excluir las ideas matemáticas poderosas en la visión cultural.

En síntesis, los marcos conceptuales sobre modelación matemática en los documentos curriculares, privilegian su continuidad en todos los niveles educativos, sin embargo, en la configuración de los estándares, este proceso solo se tiene presencia explicita finalizando la educación básica y en la educación media. Por otra parte, es posible que el proceso de modelación como propósito formativo constituya una oportunidad para el empoderamiento critico de los estudiantes como sujetos políticos, pero es necesario conectar los diseños de tareas de modelación con ideas matemáticas poderosas en la visión sociocultural. En los cuales sea posible motivar y fortalecer la sensibilidad por las necesidades del otro y de emprender la transformación aquellos modelos que vulneren algún derecho social, tal es el caso de: la salud, el salario justo, el régimen pensional, el maltrato al medioambiente o lo justo o injusto de las tarifas de los servicios públicos.

\section{Conclusiones}

Los tres nuevos factores que sustentan la inclusión de las matemáticas escolares en el currículo colombiano, tienen un direccionamiento hacia los enfoques sociocultural y crítico de la educación matemática. Bajo esta mirada se encontró: en primer lugar, que la actividad matemática privilegia la resolución de problemas de la realidad como foco de ideas matemáticas poderosas culturalmente; segundo, la inclusión de los valores democráticos vinculados con en la educación matemática ofrece la oportunidad de una participación directa de los sujetos políticos en el cuestionamiento y la transformación de prácticas de riesgo. Sin embargo, existe una marcada inclinación, sobre todo en los lineamientos curriculares, a considerar que las matemáticas conceden un poder intelectual privilegiado. Este hecho, advierte sobre el riesgo de exclusión por habilidad y del uso de unas matemáticas poderosas fundamentadas en la estructura matemática que pueden llevar a un empoderamiento intrínseco en lugar de uno crítico.

En relación con el proceso de modelación matemática incorporado como contenido curricular (MEN, 1998; MEN, 2006), y con las sugerencias de su implementación en los DBA (MEN, 2016), se evidenció que su incorporación está orientada por el uso de la modelación matemática como vehículo para introducir o desarrollar conceptos, pero una práctica curricular de los profesores sustentada en un currículo democratizante y en la modelación sociocrítica abriría la puerta al empoderamiento crítico de los sujetos. En Colombia ya existe un número considerable de investigaciones en esta vía que muestran las ventajas de la modelación, pero el problema sigue estando en el empoderamiento del profesor para realizar lecturas críticas en diferentes arenas sociales.

Como alternativas de solución, es conveniente:

- Fortalecer la formación de profesores de matemáticas en modelación matemática, tal vez como uno de los ejes del currículo.

- La inclusión de la modelación critica en programas de mejoramiento de la practica educativa de profesores en ejercicio, provocaría acciones de empoderamiento, entre ellas, la posibilidad de establecer comunidades cooperativas entre profesores y estudiantes para reflexionar alrededor de preguntas como: ¿Cómo la modelación puede promover, la participación ciudadana, la justicia social y la equidad en realidades específicas de Colombia?

- Por último, siguiendo a Valero et al., 2015; Valero (2012a; 2012b); Valero (2006); Skovsmose (1999), los DBA deben ampliar las evidencias de aprendizaje a una postura crítica que permita consolidar una línea fundamentada en los principios del empoderamiento crítico y el ejercicio de los valores democráticos. 


\section{Referencias bibliográficas}

Alrø, H., Ole, R., y Valero, P. (2010). Inter-viewing critical mathematics education." . Brill Sense, 2010. 1-9. En H. Alrø, R. Ole, \& P. Valero, Critical Mathematics Education: Past, Present and Future (págs. 1-9). Brill Sense.

Alsina, C. (2007). Si Enrique VIII tuvo 6 esposas, ¿̇uántas tuvo Enrique IV? el realismo en educación matemática y sus implicaciones docentes. Revista Iberoamericana de Educación, 43, 85-101.

Alvis, J., Aldana , E., y Solar, H. (2019). Ambientes de aprendizaje un articulador para el desarrollo de compatencias matemáticas. Revista Espacios, 40(28), 8-22. Obtenido de www.revistaespacios.com/a19v40n21/19402108.html

Araújo, Jussara. (2009). Uma abordagem socio-crítica da modelagem matemática: a perspectiva da educação matemática crítica. Alexandria revista de educação em ciências e tecnologia, 2(2), 55-68.

Barbosa, J. (2003). Modelagem Matemática na sala de aula." 27.98. Perspectiva, 27(98), 65-74.

Barbosa, J. (2006). Mathematical modelling in classroom: a socio-critical and discursive. ZDM-The international journal of mathematics education, 38(3).

Berrio, M. d. (2011). Elementos que intervienen en la construcción que hacen los estudiantes frente a los modelos matemáticos: el caso del cultivo de café. Maestría en enseñanza de las Ciencias Exactas y Naturales Tesis de maestría no publicada, Universidad Nacional de Colombia, Medellin.

Biembengut, M., y Hein, N. (2013). Mathematical modeling: Implications for teaching. En R. Lesh, P. Galbraith, C. Haines, \& A. Hurford, Modeling Students' Mathematical Modeling Competencies (págs. 481-490). Dordrecht: Springer. doi:https://doi.org/10.1007/978-94-007-6271-8

Bishop, A. (2005). Las influencias sociales en la clase de matemáticas. A. Bishop, Aproximación sociocultural a la Educación Matemática, 141. Cali: Universidad del Valle.

Burkhardt, H. (2006). Modelling in Mathematics Classrooms: reflections on past developments and the future. ZDM, 38(2), 178-195. doi:10.1007/BF02655888

Camelo, F., Perrila, W., y Mancera , G. (2016). Prácticas de modelación matemática desde una perspectiva socio crítica con estudiantes de grado undécimo. Revista Latinoamericana de Etnomatemática: Perspectivas Socioculturales de la Educación Matemática, 9(2), 67-84.

Carrillo, I. (2011). La educación en valores democráticos en los manuales de la asignatura Educación para la Ciudadanía. Revista de Educación, número extraordinario: Educación, valores y democracia , 137-159.

Chevallard, Y. (1999). El análisis de las prácticas docentes en la teoría antropológica de lo didáctico. Recherches en Didactique des Mathématiques, 19(2), 221-266.

D’Amore, B. (2005). Bases filosóficas, pedagógicas, epistemológicas y conceptuales de la Didáctica de la Matemática. Mexico: Reverté.

Da Silva, , C., y Kato, L. (2012). Quais Elementos Caracterizam uma Atividade de Modelagem Matemática na Perspectiva Sociocrítica? Bolema, 26(43), 817-838

Díaz-Barriga, Á. (2011). Competencias en educación. Corrientes de pensamiento e implicaciones para el currículo y el trabajo en el aula. Revista Iberoamericana De Educación Superior, 2(5), 3-24.

Elliot, J. (1994). La investigación-acción en educación (cuarta ed.). Madrid, España: Morata. 
Erbaş, A., Kertil, M., Çetincaya, B., Çakıroğlu, E., Alacacı, C., y Baş, S. (2014). Mathematical modeling in mathematics education: Basic concepts and different approaches. Educational Sciences: Theory \& Practice, 14(4), 1-21.

Font, V. (2002). Una organización de los programas de investigación en Didáctica de las Matemáticas. Revista Ema, 7(2), 127-170.

Gómez,, P., y Velazco, C. (2017). Complejidad y coherencia de documentos curriculares colombianos: Derechos Básicos de Aprendizaje y Mallas de Aprendizaje. Revista Colombiana de Educación, 73, 259-279.

Gravemeijer, K. (2007). Emergent Modelling as a Precursor to Mathematical Modelling. En W. Blum , P. Galbraith, H.-W. Henn, \& M. Niss, Modelling and Applications in Mathematics Education. New ICMI Study Series (Vol. 10, págs. 137-144). Boston: Springer.

Julie, C. (2002). Making relevance relevant in mathematics teacher education. En I. Vakalis, D. Hughes Hallett, D. Quinney, \& C. Kourouniotis, Compilers. Proceedings of 2nd International Conference on the Teaching of Mathematics: [ICTM-2]. New York: Wiley.

Kaiser, G., y Sriraman , B. (2006). A global survey of international perspectives on modelling in mathematics education. ZDM, 38(3), 302-310.

Kemmis, S. y McTaggart, R. (1988). Cómo planificar la Investigación-Acción. Barcelona, España: Laertes.

Lerman, S. (2000). The social turn in mathematics education research. En J. Boaler, Multiple perspectives on mathematics teaching and learning (págs. 19-44). Unid States of America: Greenwood Publishing Group, Inc.

De Educación, L. G. (1994). Ley 115 de 1994. Constitución Política de Colombia.

Ministerio de Educación Nacional. (1998b). Lineamientos de Curriculares en Matemáticas. Bogotá: Magisterio.

Ministerio de Educación Nacional MEN. (2006). Esatandares Básicos de Competencias en lenguaje, matemáticas, ciencias y ciudadanas. Bogotá: Autor.

Ministerio de Educación Nacional MEN. (2016). Derechos Básicos de Aprendizaje DBA (versión 2). Bogotá: Autor.

Niss, M., y Højgaard, T. (2011). Competencies and mathematical learning. Ideas and inspiration for the development of mathematics teaching and learning in Denmark (Vol. 485). Roskilde: IMFUFAtekst. Recuperado el 21 de Agosto de 2019

Ñaupas Paitán, H., Valdivia Dueñas, M., Palacios Vilela, J., \& Romero Delgado, H. (2014). Metodología de la investigación Cuantitativa - Cualitativa y Redacción de la Tesis (Quinta ed.). Mexico D.F: Ediciones de la U.

Orey, D., y Rosa, M. (2007). A dimensão crítica da modelagem matemática: ensinando para a eficiência sociocrítica." Revista Horizontes 25.2 (2007): 197-206. Revista Horizontes, 25(2), 197-206.

Perrenoud, P. (2007). Desarrollar la Práctica Reflexiva en el oficio de enseñar. Profesiorialización y razón pedagógica (Vol. 1). México D.F.: Graó.

Planas , P. (2010). Las teorías socioculturales en la investigación en educación matemática: reflexiones y datos bibliométricos . En S. Sociedad Española de Investigación en Educación Matemática, Investigación en educación matemática XIV (págs. pp. 163-195). Lleida: Edicions de la Universitat de Lleida . 
Sacristán, G. (1995). El currículum: Una reflexión sobre la práctica (Quinta ed., Vol. 1). Madrid: Morata.

Skovsmose, O. (1999). Hacia una filosofía de la educación matemática crítica. (P. Valero, Trad.) Bogotá: una empresa docente.

Skovsmose, O., Yasukawa, K., y Ravn, O. (2011). Scripting the world in mathematics and its ethical implications. En Philosophy of Mathematics Education Journal 26 (págs. 81-98).

Solar , H. (2009). Competencias de modelización y argumentación en interpretación de gráficas funcionales: propuesta de un modelo de competencia aplicado a un estudio de caso. Bellaterra, Barcelona. : Universitat Autónoma de Barcelona,.

Solar, H., García, B., Rojas, F., y Coronado, A. (2014). Solar, H., García, B., Rojas, F., \& Coronado, A. (2014). Propuesta de un Modelo de Competencia Matemática como articulador entre el currículo, la formación de profesores y el aprendizaje de los estudiantes. , 26(2), 33-67. Educación matemática, 26(2), 33-67.

Valero , P. (2017). El deseo de acceso y equidad en la educación matemática. Revista Colombiana de Educación, $73,99-128$.

Valero, P. (2002). Consideraciones sobre el contexto y la educación matemática para la democracia. Quadrante, 11(1), 49-59.

Valero, P. (2006). ¿De carne y hueso? La vida social y política de las competencias matemáticas. . Bogotá: MEN. En M. d. Colombia, Memorias del Foro Educativo Nacional de Colombia-Competencias matemáticas. Bogotá.

Valero, P. (2012a). Perspectivas sociopolíticas en la educación matemática. En Educación Matemática Crítica (págs. 195-216). Bogotá: Ediciones Uniandes.

Valero, P. (2012 b). La educación matemática como una red de prácticas sociales. En P. Valero, \& S. Ole, Una visión sociopolítica del aprendizaje y la enseñanza de las matemáticas (págs. 299-326). Bogotá: una empresa docente.

Valero, P., Andrade-Molina, M., y Montecino, A. (2015). Lo político en la educación matemática: de la educación matemática crítica a la política cultural de la educación matemática. Revista latinoamericana de investigación en matemática educativa, 18(3), 7-20.

Villa-Ochoa, J., Castrillón-Yepes, A., \& Sánchez-Cardona, J. (2017). Tipos de tareas de modelación para la clase de matemáticas. Espaço Plural, 18(36), 219-251.

Villa Ochoa , J., y Ruiz Vahos, H. (2009). Modelación en educación matemática: una mirada desde los lineamientos y estándares curriculares colombianos. Revista virtual Universidad Católica del Norte(27), 121. 